\title{
Experimental modeling of intergranular exchange coupling for perpendicular thin film media
}

\author{
Vincent Sokalski, ${ }^{a)}$ David E. Laughlin, and Jian-Gang Zhu \\ Data Storage Systems Center, Carnegie Mellon University, Pittsburgh, Pennsylvania 15213, USA
}

(Received 18 August 2009; accepted 21 August 2009; published online 11 September 2009)

\begin{abstract}
We present an experimental model system that enables quantitative assessment of intergranular exchange coupling in CoCrPt-oxide perpendicular magnetic recording media. A thin film structure consisting of a high coercivity CoPt unicrystal layer and a lower coercivity CoPt layer separated by a thin oxide interlayer is used to model perpendicularly magnetized grains separated by oxide grain boundaries. Exchange coupling energy between the CoPt layers was obtained for $\mathrm{SiO}_{x}$, $\mathrm{TiO}_{x}$, and $\mathrm{CrO}_{x}$ interlayers by measuring field shifts from the lower coercivity layer. $\mathrm{Cr}$ segregation in $\mathrm{CoCrPt}$ grains to grain boundaries is also modeled experimentally and found to significantly suppress exchange coupling. (C) 2009 American Institute of Physics. [doi:10.1063/1.3226638]
\end{abstract}

The recording layer in present perpendicular thin film media consists of at least two magnetic layers: a CoCrPtoxide granular layer comprising $\mathrm{CoCrPt}$ grains separated by oxide grain boundaries and a capping layer comprised of $\mathrm{CoCrPt}$ grains free of oxide grain boundaries. Ideally, the oxide grain boundaries would completely disrupt ferromagnetic exchange coupling between adjacent grains in the granular layer, while the capping layer provides spatially uniform intergranular exchange coupling. ${ }^{1}$ However, in practice, it is believed that significant intergranular exchange coupling does exist in the CoCrPt-oxide layer of present perpendicular thin film media, especially for media with very small grain sizes. $^{2,3}$

In this paper, we present an experimental method that enables one to quantitatively determine the ferromagnetic exchange coupling through an oxide layer as a function of oxide layer thickness. The model system consists of two ferromagnetic layers of a $\mathrm{Co}_{84} \mathrm{Pt}_{16}$ alloy sandwiching an oxide interlayer. Films were prepared by rf sputtering using a Leybold Heraeus Z400 sputtering system maintained at a base pressure better than $2 \times 10^{-7}$ Torr and an argon sputtering pressure fixed at 5 mTorr. A single crystal $\mathrm{MgO}(110)$ substrate is used to induce the desired epitaxial film growth [Fig. 1(a)].

The procedure described here closely follows other work done on epitaxial Co-alloy growth using MgO substrates. ${ }^{4,5}$ A $60 \mathrm{~nm}$ thick $\mathrm{Cr}$ underlayer is deposited on the $\mathrm{MgO}$ substrate. With the substrate heated to $400{ }^{\circ} \mathrm{C}$ during this deposition, the $\mathrm{Cr}$ layer grows with excellent (112) texture. After the substrate is cooled to room temperature, a $24 \mathrm{~nm}$ layer of $\mathrm{Co}_{84} \mathrm{Pt}_{16}$ is deposited on the $\mathrm{Cr}$ underlayer, which grows with

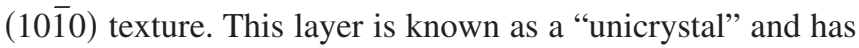
a hexagonal close-packed (HCP) structure with a single $c$-axis orientation variant lying in the plane of the film. The epitaxial relationships can be summarized as follows:

$$
\begin{aligned}
& \mathrm{Co}(10 \overline{1} 0)\|\mathrm{Cr}(112)\| \mathrm{MgO}(110), \\
& \mathrm{Co}[0001]\|\mathrm{Cr}[1 \overline{1} 0]\| \mathrm{MgO}[001] .
\end{aligned}
$$

The x-ray diffraction pattern, shown in Fig. 1(b), and plan view electron diffraction pattern, shown in Fig. 2(a), demon-

${ }^{a)}$ Electronic mail: vsokalsk@andrew.cmu.edu. strate the excellent epitaxial growth of all the layers described above. This is also verified by the in-plane hysteresis loops measured along the $\mathrm{MgO}$ [001] (easy axis) and orthogonal $\mathrm{MgO}[1 \overline{1} 0]$ (hard axis) directions in the film plane, as shown in Fig. 3(a). These loops were taken from a $50 \mathrm{~nm}$ unicrystal sample without a soft overlayer. The easy axis loop shows a coercivity of around 1000 Oe, even though the anisotropy field, as shown by the hard axis loop, is substantially higher.

After deposition of the unicrystal CoPt layer, a thin layer of oxide is deposited, followed by the deposition of another $\mathrm{Co}_{84} \mathrm{Pt}_{16}$ layer of $8 \mathrm{~nm}$ in thickness. The top CoPt layer does not show any particular texture. Figure 3(b) shows four measured hysteresis loops from this multilayer structure for a $\mathrm{TiO}_{x}$ interlayer of different thicknesses with the field applied along the $\mathrm{MgO}[001]$ direction. The interlayer exchange field is obtained by measuring the field shift, $H_{\text {shift }}$, of the hysteresis loop from the top CoPt layer. The interlayer coupling energy density is calculated according to

$$
\sigma=H_{\text {shift }} M_{s, \text { soft }} \delta_{\text {soft }},
$$

where $M_{s, \text { soft }}$ and $\delta_{\text {soft }}$ are the saturation magnetization (1100 emu/cc) and the thickness $(8 \mathrm{~nm})$ of the softer top $\mathrm{CoPt}$ layer, respectively.

For each oxide, there is a particular thickness at which point the top and bottom layers have the same reversal field and measuring a shift in the minor loop becomes impossible [e.g., $11 \AA$; Fig. 3(b)]. However, the coupling energy density can still be estimated from an observed decrease in the reversal field of the major loop following the method of van
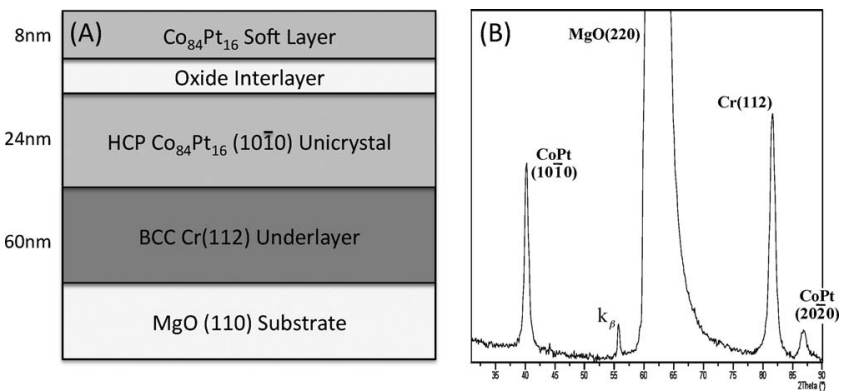

FIG. 1. (a) Structure of experimental model system. (b) $\theta / 2 \theta$ XRD pattern of a $\mathrm{Co}_{84} \mathrm{Pt}_{16}$ unicrystal. 

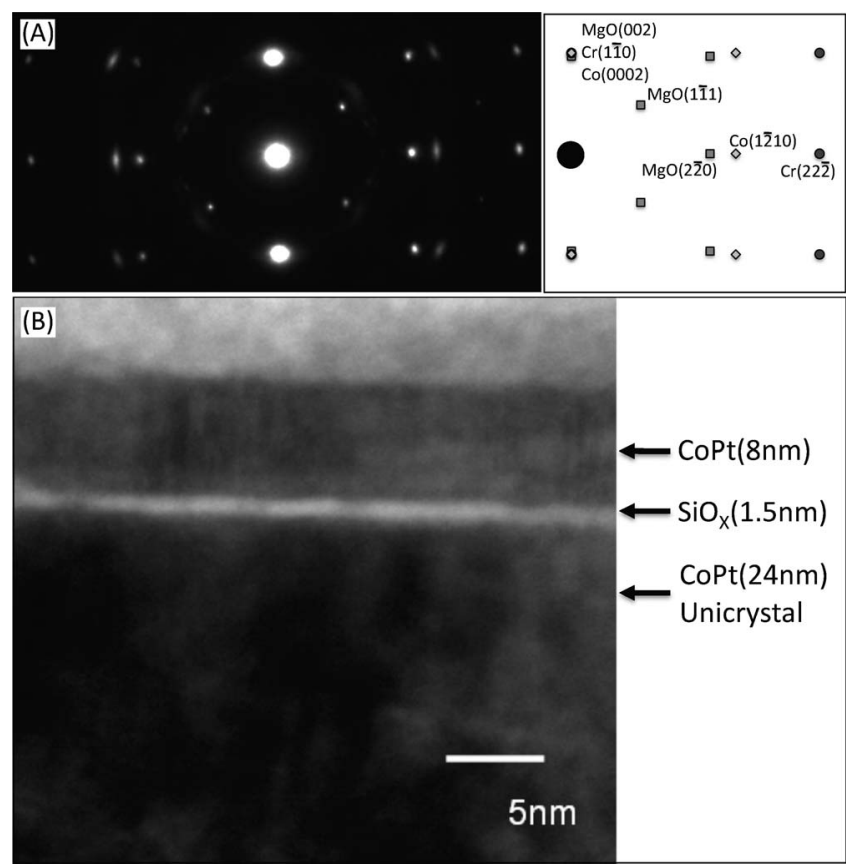

FIG. 2. (a) Plan view unicrystal electron diffraction pattern and predicted pattern w/indexing (right half shown only). (b) Cross-section TEM image showing CoPt layers with $1.5 \mathrm{~nm} \mathrm{SiO}_{x}$ interlayer.

der Heijden et al. ${ }^{6}$ This decrease is a consequence of the exchange coupling felt by the unicrystal from the soft layer and is expressed as

$$
\sigma=\left(H_{r, 0}-H_{r}\right) M_{S, \text { hard }} \delta_{\text {hard }},
$$

where $H_{r, 0}$ is the intrinsic reversal field (i.e., the coercivity) of the unicrystal layer without interlayer coupling and $H_{r}$ is the reversal field with interlayer coupling.

Cross-section transmission electron microscope (TEM) micrographs show that the oxide interlayer is reasonably smooth and has no obvious discontinuities for the thicknesses studied. Figure 2(b) shows a representative crosssection for a $\mathrm{SiO}_{x}$ interlayer of $1.5 \mathrm{~nm}$ thickness.

Figure 4 shows the interlayer coupling energy density as a function of the oxide layer thickness for three different oxides: $\mathrm{CrO}_{x}$ (target composition: $\left.\mathrm{Cr}_{2} \mathrm{O}_{3}\right), \mathrm{TiO}_{x}\left(\mathrm{TiO}_{2}\right)$, and $\mathrm{SiO}_{x}\left(\mathrm{SiO}_{2}\right)$. The symbols are the actual measurement data and the curves are fitting curves with the following thickness dependence:

$$
\sigma=A \exp \left(-\frac{\delta_{\text {oxide }}}{B}\right)+C \cdot \delta_{\text {oxide }}+D,
$$

where $\delta_{\text {oxide }}$ is the oxide layer thickness and $A, B, C$, and $D$ are all fitting parameters with typical values on the order of
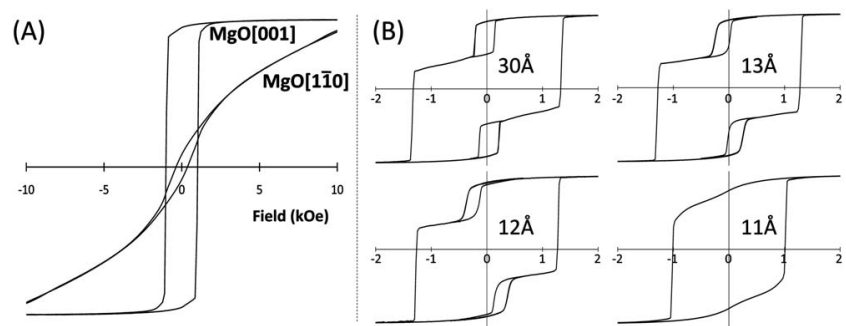

FIG. 3. (a) Unicrystal in-plane hysteresis loops measured along orthogonal $\mathrm{MgO}$ directions. (b) Example hysteresis loops showing exchange coupling with varying $\mathrm{TiO}_{x}$ interlayer thickness.
$10^{5} \mathrm{ergs} / \mathrm{cm}^{2}, 1 \AA, 10^{4} \mathrm{ergs} / \mathrm{cm}^{3}$, and $0.1 \mathrm{ergs} / \mathrm{cm}^{2}$, respectively. For all three cases, the coupling is ferromagnetic in nature.

The nonzero offset of the coupling energy is likely due to magnetostatic "orange peel" coupling between the two ferromagnetic layers. ${ }^{7,8}$ However, the exponential increase at lower thickness cannot be explained by this magnetostatic coupling mechanism for any plausible interfacial roughness parameters. Therefore, it is reasonable to attribute the sharp increase in energy density to ferromagnetic exchange through the oxide interlayer. While the particular mechanism that explains this ferromagnetic exchange coupling remains unclear, a number of other reports have attributed similar trends in coupling energy to discontinuities, or "pinholes," in the interlayer. ${ }^{6,9}, 10$ Although detection of pinholes would require a more rigorous study of the oxide interlayer morphology, our preliminary TEM work does not show any obvious discontinuities. It is important to recognize that regardless of the mechanism, the trend is highly dependent on the type of oxide material that is used and is likely related to fundamental properties of the $\mathrm{CoPt} /$ oxide interface.

If we define the critical thickness to be the point at which the coupling energy density reaches $0.2 \mathrm{ergs} / \mathrm{cm}^{2}$, the critical thickness for $\mathrm{CrO}_{x}$ is at $1.2 \mathrm{~nm}, \mathrm{TiO}_{x}$ at $1.25 \mathrm{~nm}$, and $\mathrm{SiO}_{x}$ at $1.8 \mathrm{~nm}$. The deposition conditions were maintained the same for each layer, including the oxide layers, within this set of presented data. In addition, the oxide deposition conditions were varied for a given oxide by changing the sputtering power, temperature, and deposition rate. It was found that the interlayer exchange coupling was largely independent of these parameters. We conclude that the coupling strength, as a function of oxide layer thickness, is very much a characteristic of the oxide material. It is interesting to note that significant exchange coupling can occur through a $\mathrm{SiO}_{x}$ layer as thick as thick $1.8 \mathrm{~nm}$, even though the layer appears to be continuous.

Magnetic grains in present perpendicular thin film media are actually composed of a CoCrPt alloy (rather than CoPt alloy used in our experiment), and experimental studies have found significant $\mathrm{Cr}$ segregation to grain boundaries. ${ }^{11-13} \mathrm{It}$ has long been suggested that a $\mathrm{Cr}$-rich $\mathrm{CoCr}$ grain boundary could result in a significant disruption of intergranular exchange coupling. ${ }^{14}$ To mimic such grain boundary conditions in today's perpendicular thin film media, i.e., to study the impact of Cr segregation on intergranular exchange coupling, we performed the following addition to the experiments: very thin $\mathrm{Cr}$ layers, $1.0-1.5 \AA$, were deposited prior to, as well as after, the deposition of the oxide layer. Such interfacial "dusting" of pure $\mathrm{Cr}$ was performed for both $\mathrm{TiO}_{x}$ and $\mathrm{SiO}_{x}$ layers, the two most commonly used oxide materials in present commercial perpendicular media. Figures 5(a) and 5(b) show the measured interlayer coupling energy densities for the Cr dusting on $\mathrm{SiO}_{x}$ and $\mathrm{TiO}_{x}$ layers, respectively. In the case of a $\mathrm{SiO}_{x}$ layer, with the $\mathrm{Cr}$ dusting, the coupling versus oxide layer thickness curve shifts by almost $8 \AA$. The thickness shift is significantly greater than the added $\mathrm{Cr}$ thickness. In the case of $\mathrm{TiO}_{x}$, the shift is significantly smaller as compared with the $\mathrm{SiO}_{x}$ interlayer. Note that the resulting critical thicknesses of the two oxide layers after the $\mathrm{Cr}$ dusting become very similar, even though they are very different without the $\mathrm{Cr}$ dusting.

The observed effect of interfacial $\mathrm{Cr}$ dusting could be due to $\mathrm{Cr}$ diffusion into the $\mathrm{CoPt}$ layers. $\mathrm{Cr} / \mathrm{CoPt}$ interfacial 


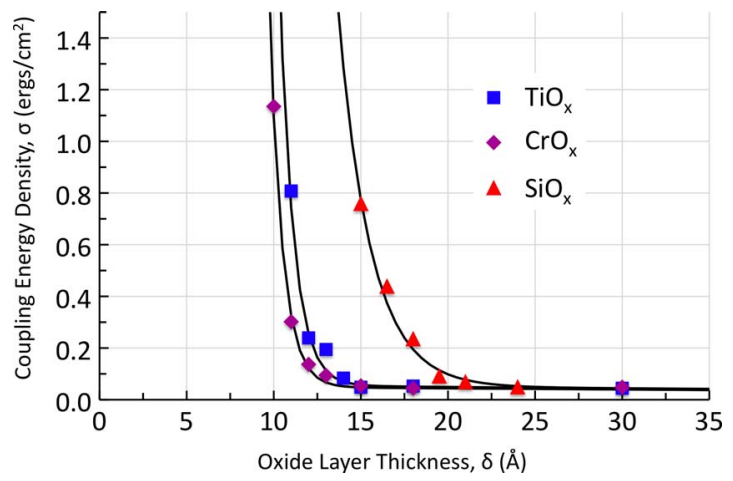

FIG. 4. (Color online) Coupling energy density as a function of oxide thickness for $\mathrm{SiO}_{x}, \mathrm{TiO}_{x}$, and $\mathrm{CrO}_{x}$.

diffusion could very well lead to the formation of a thin $\mathrm{CoCrPt}$ layer with large enough $\mathrm{Cr}$ concentration to make the layer paramagnetic instead. The formation of such a layer should result in significant reduction of the interlayer exchange coupling. The measurement data shown in Fig. 5 suggest that an oxide grain boundary thicker than $1 \mathrm{~nm}$ could significantly suppress ferromagnetic exchange coupling from the adjacent grains.

If the characteristic dependence between the exchange coupling strength and the thickness of an oxide interlayer can be applied to magnetic grains separated by grain boundaries made of the same oxide, any adjacent magnetic grains with grain boundaries thinner than the critical thickness would experience significant intergranular exchange coupling. More importantly, variation in grain boundary thickness below the critical thickness will yield a substantial variation in the intergranular coupling throughout the film. Such variation in the intergranular exchange coupling over the media will undoubtedly lead to a transition noise increase since large spatial fluctuations in the exchange fields will yield significant shifts in transition boundaries locally. These spatial fluctuations can only be eliminated if the oxide grain boundary thickness distribution is beyond the critical thickness. The optimum intergranular exchange coupling strength can be achieved by tuning the thickness ratio between the CoCrPtoxide and the capping layers. Any distribution of oxide grain boundary thickness below the critical thickness will make spatially uniform intergranular exchange coupling impossible within the CoCrPt-oxide layer itself.

In summary, we have developed an experimental model system to quantitatively assess the intergranular exchange coupling through oxide grain boundaries in present perpendicular thin film recording media. For all the oxides studied here, the ferromagnetic exchange coupling shows an exponential dependence on the oxide layer thickness when the thickness is below a critical value. It is found that this critical thickness is a strong function of the oxide and can be decreased significantly by interfacial "dusting" of chromium, especially for $\mathrm{SiO}_{x}$. Although the exact formation process of the oxide grain boundaries could be different from the one for the oxide layer formed by planar deposition in our model system, there is experimental evidence that shows a strong dependence of intergranular exchange coupling on the particular oxide material much like what has been shown by the results presented here. ${ }^{3,15}$ The modeled experimental system

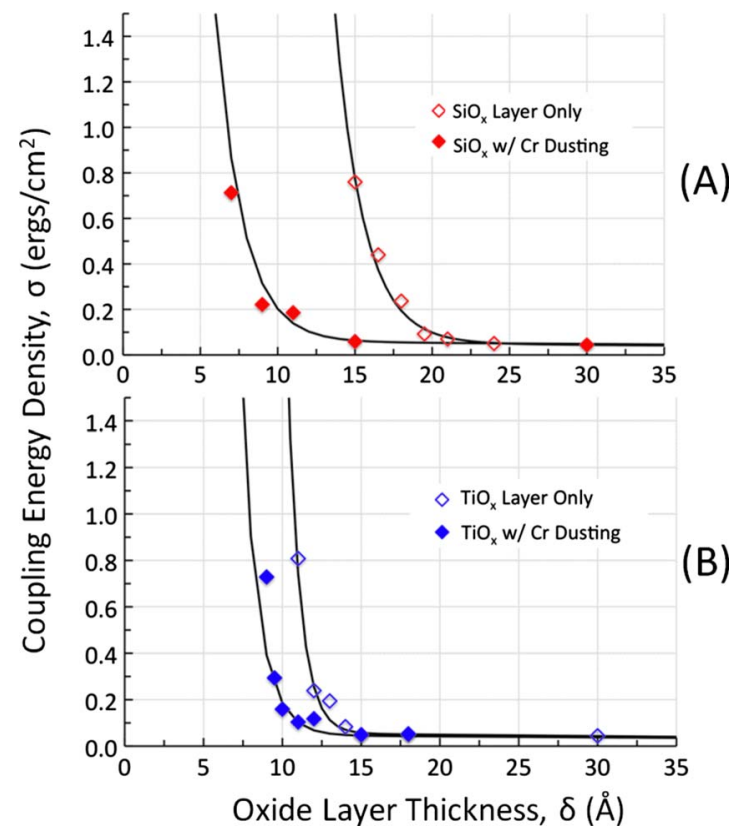

FIG. 5. (Color online) Comparison of coupling energy density with and without Cr dusting for (a) $\mathrm{SiO}_{x}$ and (b) $\mathrm{TiO}_{x}$.

should also enable studies on various other types of intergranular materials in terms of their ability to exchange decouple independent of their capability to form well defined grain boundaries for a given fabrication process.

The authors would like to thank Showa Denko (SDK) and the Data Storage Systems Center (DSSC) of Carnegie Mellon University for financial support.

${ }^{1}$ G. Choe, M. Zheng, B. R. Acharya, E. N. Abarra, and J. N. Zhou, IEEE Trans. Magn. 41, 3172 (2005).

${ }^{2}$ R. Araki, Y. Takahashi, I. Takekuma, and S. Narishige, IEEE Trans. Magn. 44, 3496 (2008).

${ }^{3}$ I. Tamai, R. Araki, and K. Tanahashi, IEEE Trans. Magn. 44, 3492 (2008).

${ }^{4}$ A. Nakamura and M. Futamoto, Jpn. J. Appl. Phys., Part 1 32, L1410 (1993).

${ }^{5}$ A. Nakamura, M. Koguchi, and M. Futamoto, Jpn. J. Appl. Phys., Part 1 34, 2307 (1995).

${ }^{6}$ P. A. A. van der Heijden, P. J. H. Bloemen, J. M. Metselaar, R. M. Wolf, J. M. Gaines, J. T. W. M. v. Eemeren, P. J. van der Zaag, and W. J. M. d. Jonge, Phys. Rev. B 55, 11569 (1997).

${ }^{7}$ L. Néel, C. R. Acad. Sci. 255, 1676 (1962).

${ }^{8}$ B. D. Schrag, A. Anguelouch, S. Ingvarsson, G. Xiao, Y. Lu, P. L. Trouilloud, A. Gupta, R. A. Wanner, W. J. Gallagher, P. M. Rice, and S. S. P. Parkin, Appl. Phys. Lett. 77, 2373 (2000).

${ }^{9}$ P. J. van der Zaag, P. J. H. Bloemen, J. M. Gaines, R. M. Wolf, P. A. A. d. Heijden, R. J. M. d. Veerdonk, and W. J. M. d. Jonge, J. Magn. Magn. Mater. 211, 301 (2000)

${ }^{10}$ C. L. Platt, M. R. McCartney, F. T. Parker, and A. E. Berkowitz, Phys. Rev. B 61, 9633 (2000).

${ }^{11}$ G. Choe, M. Zheng, E. N. Abarra, B. G. Demczyk, J. N. Zhou, B. R. Acharya, and K. E. Johnson, J. Magn. Magn. Mater. 287, 159 (2005).

${ }^{12}$ J.-G. Zhu, H. Yuan, S. Park, T. Nuhfer, and D. E. Laughlin, IEEE Trans. Magn. 45, 911 (2009).

${ }^{13}$ D. E. Laughlin, N. T. Nuhfer, S. Park, H. Yuan, and J.-G. Zhu, J. Appl. Phys. 105, $07 \mathrm{~B} 739$ (2009).

${ }^{14}$ J. Nakai, E. Kusumoto, M. Kuwabara, T. Miyamoto, M. R. Visokay, K. Yoshikawa, and K. Itayama, IEEE Trans. Magn. 30, 3969 (1994).

${ }^{15}$ G. Choe, A. Roy, Z. Yang, B. R. Acharya, and E. N. Abarra, IEEE Trans. Magn. 42, 2327 (2006). 